\title{
Review
}

\section{Recent Technical Developments of Immunomolecular Histochemistry and its Applications to Diagnostic Pathology}

\author{
R. Yoshiyuki Osamura \\ Department of Pathology, Tokai University School of Medicine, Boseidai, Isehara-city, Kanagawa 259-1193
}

Received for publication March 5, 1999

\begin{abstract}
Immuno-molecular techniques specifically covers to the recent development of immunohistochemistry $(\mathrm{IHC})$ and in situ hybridization (ISH). Particularly, in this review article, IHC emphasizes heat induced epitope retrieval (HIER) and tyramide (catalyzed) signal amplification (TSA or CSA) and ISH; focuses on peptide nucleic acid (PNA). The TSA (or

(SA) is the reaction with marked amplification and has been useful in detecting trivial amounts of antigens. It is also applicable to ISH. PNA technology has been reported to be a stable detection method for RNA such as EBER. These methods have contributed to diagnostic pathology in the aspects of achievement and control of quality.
\end{abstract}

Key words: Immunohistochemistry, In situ hybridization, Peptide nucleic acid (PNA), Tyramide signal amplification, Antigen retrieval

\section{Introduction}

Histochemistry, especially immunohistochemistry, has contributed to diagnostic pathology in diagnostic accuracy and quality control. Recent technical developments have resulted in markedly improved sensitivity. These techniques include dextran polymer histochemistry and tyramide (catalyzed) signal amplification. The other major technical achievement that should be emphasized is the detection of nucleotides by in situ hybridization (ISH). The method has been simplified and made more applicable using non-radioisotopic probes. ISH has been benefited from the new technology, of peptide nucleic acid (PNA). This review article describes recent developments and the application of histochemisty to diagnostic pathology.

\section{Immunohistochemistry}

Two major aspects of improvements in immunohistochemical staining deserve special attention, heat induced epitope retrieval (HIER) [2] and amplification of sensitivity [3]. For HIER, most of the commercially availa-

Correspondence to: R. Yoshiyuki Osamura, M.D., Department of Pathology, Tokai University School of Medicine, Boseidai, Isehara-city, Kanagawa 259-1193, Japan. ble antibodies take advantage of this method. $\mathrm{pH}$ and heat are the major factors in improving the restoration of antigenicity. For amplification, tyramide (catalyzed) signal amplification (TSA, or CSA) is of particular importance. Sanno et al. [3] has reported that the CSA system enabled the primary antibodies to be diluted 100 times more than the ABC method. This technique is expected to detect trivial amount of antigens in tissue sections. One drawback of this method in routine diagnostic use is that it requires 8 steps during the staining procedures. The other more simple amplification technique uses a polymer molecule on which antibodies and peroxidase molecules are conjugated. Theoretically, by this technology, many peroxidase molecules can react with an antigen molecule in the tissue sections. This method can be applied as a modified method of peroxidase-labeled antibody method (by Nakane). The combination of HIER technique and the amplification method can detect trivial amounts of substances which are sometimes essential in differential diagnosis.

\section{In Situ Hybridization (ISH)}

The usage of non-radioisotopic labeling of probes has made this method applicable in routine diagnostic laboratories and ISH has prevailed as a stable method to detect 
DNA and RNA [2]. Biotin or digoxigenin-labeled oligonucleotides have been frequently used, and pre-labeled probe kits have been made available commercially. For diagnostic purposes, ISH is mandatory and has been frequently used for the detection of viruses. Particularly, carcinogenic subtypes of human papilloma virus $16 / 18$, $31 / 33 / 51$ could be detected only by ISH on tissue sections or cytologic smears. ISH has been also frequently used for detection of Epstein-Barr virus (EBV) in various neoplastic lesions. For particular situations, the detection of mRNA could be also useful in differential diagnosis. The detection of kappa chain mRNA and lambda chain mRNA is especially useful for the evaluation of monoclonality in plasma cell proliferation. Although IHC shows background staining by the presence of serum proteins in the interstitium, ISH can show a more clear-cut distinction between kappa mRNA or lambda mRNA in the cytoplasm.

Recently, new technology for ISH has been developed by using peptide nucleic acid (PNA) [4]. This technique uses nucleotides conjugated to peptides, in which no particular attention for RNase should be necessary. From our experience, in EBV-associated gastric carcinoma, PNA method could detect EBER more intensely and more stably than the conventional ISH technique.

\section{Summary}

With the recent technical developments, immunomolecular histochemistry, which covers immunohistochemistry and in situ hybrdization, is expected to play major ancillary roles for diagnostic pathology, in both histology and cytology.

\section{References}

1. Kuhn, H., Demidov, V. V., Frank-Kamenetskii, M. D. and Neilsen, P. E.: Kinetic sequence descrimination of cationicbis-PNAs upon targeting of double-stranded DNA. Nucleic Acid. Res. 26; 582-587, 1998.

2. Osamura, R. Y.: Electron microscopic basis for applications of immuno-histochemistry (IHC) and in situ hybridization (ISH) to diagnostic pathology. Acta Histochem. Cytochem. 28; 187$189,1995$.

3. Sanno, N., Osamura, R.Y. and Teramoto, A.: Application of catalyzed signal amplification (CSA) in immunodetection of gonadotropin subunits in clinically nonfunctioning pituitary adenomas. Am. J. Clin. Pathol. 106; 16-21, 1996.

4. Serizawa, A., Kawai, K. and Osamura, R. Y.: Application of peptide nucleic acid (PNA) to detect Epstein-Barr virus in tissue sections. Laboratory Medicine 42; 1013-1016, 1998. (in Japanese) 